\title{
NMAAP1 Expressed in BCG-Activated Macrophage Promotes M1 Macrophage Polarization
}

\author{
Qihui Liu', Yuan Tian', Xiangfeng Zhao'2, Haifeng Jing', Qi Xie', Peng Lí', Dong Lí', Dongmei Yan ${ }^{1, *}$, and \\ Xun Zhu,
}

\begin{abstract}
Macrophages are divided into two subpopulations: classically activated macrophages (M1) and alternatively activated macrophages (M2). BCG (Bacilli Calmette-Guérin) activates disabled naïve macrophages to M1 macrophages, which act as inflammatory, microbicidal and tumoricidal cells through cell-cell contact and/or the release of soluble factors. Various transcription factors and signaling pathways are involved in the regulation of macrophage activation and polarization. We discovered that BCG-activated macrophages (BAM) expressed a new molecule, and we named it Novel Macrophage Activated Associated Protein 1 (NMAAP1).

The current study found that the overexpression of NMAAP1 in macrophages results in M1 polarization with increased expression levels of M1 genes, such as inducible nitric oxide synthase (iNOS), tumor necrosis factor alpha (TNF- $\alpha$ ), Interleukin 6 (IL-6), Interleukin 12 (IL-12), Monocyte chemoattractant protein-1 (MCP-1) and Interleukin-1 beta (IL-1 $\beta$ ), and decreased expression of some M2 genes, such as Kruppel-like factor 4 (KLF4) and suppressor of cytokine signaling 1 (SOCS1), but not other M2 genes, including arginase-1 (Arg-1), Interleukin (IL-10), transforming growth factor beta (TGF- $\beta$ ) and found in inflammatory zone 1 (Fizz1). Moreover, NMAAP1 overexpression in the RAW264.7 cell line increased cytotoxicity against MCA207 tumor cells, which depends on increased inflammatory cytokines rather than cell-cell contact. NMAAP1 also substantially enhanced the phagocytic ability of macrophages, which implies that NMAAP1 promoted macrophage adhesive and clearance activities. Our results indicate that NMAAP1 is an essential molecule that modulates macrophages phenotype and plays an important role in macrophage tumoricidal functions.
\end{abstract}

\footnotetext{
${ }^{1}$ Department of Immunology, College of Basic Medical Sciences, Jilin University, Changchun 130021, China, 'Department of Immunology, Faculty of Basic Medical Sciences, Guilin Medical University, Guilin 541004, China

*Correspondence: dmyan@jlu.edu.cn (DY); zxunzhux@vip.sohu.com $(X Z)$
}

Received 11 May, 2015; revised 9 July, 2015; accepted 13 July, 2015; published online 2 October, 2015

Keywords: differentiation, M1, macrophage, NMAAP1, tumor

\section{INTRODUCTION}

Activated macrophages produce various cytokines, chemokines, and enzymes (Chan et al., 2011), and exhibit diverse and opposing functions, including pro- and anti-inflammatory responses, tissue repair and destruction, and tumoricidal, proangiogenesis and pro-tumoral functions (Labonte et al., 2014; Villalta et al., 2009; Wang et al., 2014). The paradoxical plasticity of macrophage function is closely related to the polarized activation state.

Heterogenic macrophages are divided into two subpopulations: classically activated macrophages (M1) and alternatively activated macrophages (M2). LPS and IFN gamma activate classical or M1 macrophages, which exhibit the following characteristics: high endocytic and phagocytic capacities; high production of interleukin-12 (IL-12), interleukin-23 (IL-23) and toxic intermediates (e.g., nitric oxide and reactive oxygen intermediates); great antigen presentation abilities; and high antimicrobial and anti-proliferative activities (Sica and Mantovani, 2012). Therefore, M1 macrophages are generally considered potent effector cells that exhibit anti-microbial and tumoricidal abilities and secrete large amounts of pro-inflammatory cytokines (Sica et al., 2014). In contrast, interleukin-4 (IL-4), interleukin-13 (IL-13), glucocorticoids, interleukin-10 (IL-10) or immunoglobulin complexes activate alternatively activated or M2 macrophages. M2 cells produce small amounts of inflammatory cytokines (e.g., IL-12 and IL-23), promote tissue remodeling and repair, contribute to angiogenesis and tumor progression, and exhibit low antigen presentation capacity (Gordon et al., 2010). These seemingly opposing effects may be explained by the existence of phenotypically and functionally heterogeneous macrophages. Several molecules, including miR223, suppressor of cytokine signaling 3 (SOCS3), Kruppel-like factor 4 (KLF4) and peroxisome proliferator-activated receptors $\gamma$ (PPAR $\gamma$ ), and cells, such as Th1, Th2, Tregs and B cells, regulate macrophage polarization and contribute to different macrophage functions (Wang et al., 2014; Wong et al., 2010).

Macrophages obtain surprisingly high tumoricidal activities after activation by select stimuli. Bacilli Calmette-Guérin (BCG) is a strong stimulus that is used for the treatment of superficial bladder cancer (Huang et al., 2015; Liu et al., 2014). However, the mechanism of action of BCG is not clear. Several studies suggest that the tumoricidal effect is related to the ability of BCG to activate macrophages (Lodillinsky et al., 2010; Luo et al., 2006; Svatek et al., 2015; Thompson et al., 2015). Stimulation of macrophages with BCG in vitro induces cytotoxicity 
against bladder cancer cells via interleukin-6 (IL-6), interleukin12 (IL-12), and tumor necrosis factor alpha (TNF- $\alpha$ ) secretion, and this activation process is inhibited by IL-10 and direct contact with cancer cells (Benoit et al., 2008; Svatek et al., 2015). The cytotoxicity of BCG-activated macrophages requires direct cell-to-cell contact and soluble factors, such as IL-6, Interferon gamma (FN- $\gamma$ ) and TNF- $\alpha$. Neutralizing endogenous cytokines, including IL-12, IL-18, IFN- $\gamma$ and TNF- $\alpha$, reduce the cytotoxicity of BCG-activated macrophage by $38 \%, 22 \%, 15 \%$ and $94 \%$, respectively (Luo et al., 2006).

Novel macrophage activated-associated protein 1 (NMAAP1, accession number: NP 001001738) was identified using capillary liquid chromatography tandem-mass spectrometry (LCMS/MS) in BCG-activated macrophage (Zhang et al., 2007). NMAAP1 is a novel membrane-associated protein in BCGactivated macrophages that contains a partial male abnormal 21 (MAB-21) domain, and it is expressed in a wide range of tissues (Kang et al., 2010). MAB-21 is a conserved sequence that acts as part of the mechanism required for correct cell fate choice, and the MAB-21 gene in vertebrates exhibits an amino acid homology up to $90 \%$ (Baldessari et al., 2004; Yamada et al., 2004; Zhao et al., 2011). DANGER1A has an MAB-21 domain that binds the inositol trisphosphate receptor (IP3R) to promote the differentiation and outgrowth of neuronal processes (Ho et al., 2001). Whether NMAAP1 has the same effect as DANGER1A is not known.

This study investigated the relationship between macrophage polarization state and NMAAP1 expression after BCG treatment in a mouse model to clarify the physiological function of NMAAP1 in BCG-activated macrophage polarization. NMAAP1 overexpression in RAW264.7 cells was established to investigate the effect of NMAAP1 on macrophage phenotype, phagocytic ability and tumoricidal activities. Our results demonstrate that NMAAP1 is a modulatory protein in macrophage polarization and cytotoxicity.

\section{MATERIALS AND METHODS}

\section{Cells and animals}

RAW264.7 cells (murine macrophage cell line ATCC TIB-71) and transfected RAW264.7 cells were cultured in Dulbecco's Modified Eagle's Medium (DMEM) with 10\% heat-inactivated fetal bovine serum (Sigma, USA), $100 \mu \mathrm{g} / \mathrm{ml}$ streptomycin and $100 \mathrm{IU} / \mathrm{ml}$ penicillin (Invitrogen). Cells were maintained at $37^{\circ} \mathrm{C}$ in a $5 \% \mathrm{CO}_{2}$ incubator. The MCA207 cell line was kindly provided by Prof. Kangla Tsung (Stanford University, USA). This cell line is a methylcholan-threne-induced transplantable tumor in C57BL/6 mice, and these cells were maintained in the same conditions. ICR mice were purchased from the Experimental Animal Center of Jilin University. All mice were housed under specific pathogen-free conditions in the animal facilities of Jilin University. The Institutional Animal Care and Use Committee of Jilin University approved the experimental procedures.

\section{Transfection}

RAW264.7 cells were seeded at $2 \times 10^{5}$ per well in a 24-well plate and grown until $70-80 \%$ confluency before transfection. pIRES2-EGFP/NMAAP1 or pIRES2-EGFP/CONTROL plasmids were directly transfected into the cells using Lipofectamine $^{\mathrm{TM}} 2000$ Transfection Reagent (Invitrogen, USA) according to the manufacturer's protocol. Fluorescein-labeled pIRES2EGFP/NMAAP1 or pIRES2-EGFP/CONTROL transfected cells were examined under fluorescence optics to determine transfection efficiency after 24 and 48 h. 3-5\% and 7-8\% initial trans- fection efficiency was observed in RAW264.7 cells 24 and $48 \mathrm{~h}$ post-transfection, respectively. Selection was performed $72 \mathrm{~h}$ later using media that contained G418 $(400 \mu \mathrm{g} / \mathrm{ml})$. Culture continued for 14 days to generate stable transfectants, and G418-resistant clones were isolated. GFP signals from transfected RAW264.7 cells were assessed using fluorescence microscopy. Clones were further expanded and analyzed usingby regular PCR and Western blotting. Transfected cells were used for subsequent experiments.

\section{BCG treatment and peritoneal macrophage preparation}

ICR mice ( $n=6 /$ group/treatment/experiment) were injected intraperitoneally with $2 \mathrm{mg} \mathrm{BCG}$ (Shanghai Institute of Biological Products) or PBS on day 2 and day 10. Peritoneal exudate cells were harvested from these mice, and peritoneal macrophages were purified on days 4,8 , and 12 by washing cells from peritoneal cavity lavage with $5 \mathrm{ml}$ of ice-cold, sterile PBS, according to a previously described protocol (Park et al., 2014). The cells were distributed in DMEM supplemented with $10 \%$ FBS in six-well tissue culture plates $\left(5 \times 10^{6}\right.$ cells $\left./ \mathrm{ml}\right)$ and incubated for $4 \mathrm{~h}$. Non-adherent cells were removed by washing twice with Opti-MEM at $37^{\circ} \mathrm{C}$ in a $5 \% \mathrm{CO}_{2}$ incubator.

\section{Reverse transcription and quantitative real-time $P C R$ (qRT-PCR)}

Total RNA was directly isolated from adherent macrophages using Trizol reagent (Invitrogen, USA), according to the manufacturer's protocol. RNA quantity and quality were determined using an absorbance microplate reader TQuant (BioTEK, USA). cDNA for reverse transcription was prepared from $2 \mu \mathrm{g}$ of total RNA using oligo(dT) primers and reverse transcriptase M-MLV in a total volume of $20 \mu \mathrm{l}$, according to the manufacturer's instructions (Takara, Japan). The resulting CDNA was used for real-time PCR for NMAAP1, iNOSII and cytokines (ARG-1, TNF- $\alpha$, IL-10, and TGF- $\beta$ ) using an ABI PRISM 7300 sequence detection system (Applied Biosystems, USA). Quantitative PCR was performed using SYBR green PCR master mix (Applied Biosystem, USA). Expression of each gene was normalized to the expression of the housekeeping gene, glyceraldehyde-3phosphate dehydrogenase (GAPDH). The following forward and reverse specific primer sequences were used:

NMAAP1: Forward 5'-CCTTCCCCTGCCCAATA-3' Reverse 5'-TCCACGAAACCTTCCACA-3'

iNOSII: Forward 5'-ATGGCAACATCAGGTCGG-3' Reverse 5'-GCACAACTGGGTGAACTCC-3'

IL12p40: Forward 5'- ACTCACATCTGCTGCTCCAC-3' Reverse 5'- CGTCCGGAGTAATTTGGTGC-3'

IL-6: Forward 5'-TAGTCCTTCCTACCCCAATTT-3' Reverse 5'-TTGGTCCTTAGCCACTCCTTC-3'

KLF4: Forward 5'-TATACATTCCGCCACAGCAG-3' Reverse 5'-CGCCTCTTGCTTAATCTTGG-3'

MCP-1: Forward 5'-TTAAAAACCTGGATCGGAACC-3' Reverse 5'-GCATTAGCTTCAGATTTACGG-3'

Fizz1: Forward 5'-CCCTTCTCATCTGCATCT-3' Reverse 5'-CTGGATTGGCAAGAAGTTCC-3'

SOCS1: Forward 5'-CTGCGGCTTCTATTGGGGAC-3' Reverse 5'-AAAAGGCAGTCGAAGTCTCG-3'

SOCS3: Forward 5'-CCCTTGCAGTTCTAAGTTCAA-3' Reverse 5'-ACCTTTGACAAGCGGACTCTC-3'

ARG-1: Forward 5'-CAGTCTGGCAGTTGGAAGC-3' Reverse 5'-GGTTGTCAGGGGAGTGTTG-3'

TNF- $\alpha$ : Forward 5'-ACTGAACTTCGGGGTGATCG-3' Reverse 5'-CCACTTGGTGGTTTGCTACG-3' 
NMAAP1 Promotes M1 Macrophage Polarization

Qihui Liu et al.

\section{IL-10: Forward 5'-GTTGCCAAGCCTTATCGG-3'} Reverse 5'-GCTCTTATTTTCACAGGGGAG-3'

TGF- $\beta$ : Forward 5'-GAGGCGGTGCTCGCTTTGTA-3' Reverse 5'-CGTTGTTGCGGTCCACCATTA-3'

GAPDH: Forward 5'-GACTTCAACAGCAACTCCCACTC-3' Reverse 5'-TAGCCGTATTCATTGTCATACCAG-3'

Each reaction for real-time quantitative PCR contained $1 x$ SYBR Green PCR master mixture (Power SYBR Green PCR Master Mix; Applied Biosystems, UK), 10 pmol of each primer, and $1 \mu \mathrm{l}$ of cDNA in a final volume of $20 \mu \mathrm{l}$. The following reaction conditions were used: initial activation step $\left(5 \mathrm{~min}\right.$ at $\left.95^{\circ} \mathrm{C}\right)$ and cycling step (denaturation for $30 \mathrm{~s}$ at $94^{\circ} \mathrm{C}$, annealing for $30 \mathrm{~s}$ at $60^{\circ} \mathrm{C}$ and extension for $1 \mathrm{~min}$ at $72^{\circ} \mathrm{C} \times 40$ cycles) followed by melt curve analysis. Detection of the dequenched probe, calculation of threshold cycles (Ct values), and further data analyses were performed using the Sequence Detector software. Relative changes in NMAAP1, iNOSII and cytokines (ARG-1, TNF- $\alpha$, IL-10, and TGF- $\beta$ ) mRNA expression were compared with control, normalized to GAPDH, and were quantified using the 2-( $\Delta \Delta \mathrm{Ct})$ method. Therefore, all values for experimental samples are expressed as fold differences between the sample mRNA and the calibrator (GAPDH) mRNA.

\section{Flow cytometric analysis}

Cells were washed twice in phosphate-buffered saline, counted, and re-suspended in FACS buffer (3\% FBS in PBS containing $0.01 \% \mathrm{NaN}_{3}$ ). Cells $\left(1 \times 10^{6}\right.$ cells/samples $)$ for phenotypic analyses were stained using the indicated fluorochromeconjugated antibody for 30 min on ice. Cells were washed with FACS buffer, and appropriate isotype controls were used in all cases. Typical forward- and side-scatter gates for flow cytometric analyses were set to exclude dead and aggregated cells. A total of $10^{4}$ events in the gate were collected and analyzed using Accuri $\mathrm{C} 6$ and software (BD Biosciences, USA). The anti-CD23, anti-CD16/32 and anti-F4/80 antibodies (Abs) used in flow cytometric analyses were obtained from eBioscience
(USA).

\section{Cytokines measurement using ELISA}

Blood was extracted from mouse eye sockets. Serum was prepared using routine methods and stored in a $-80^{\circ} \mathrm{C}$ refrigerator for subsequent tests. Serum and supernatant cytokine levels of were measured using an enzyme-linked immunoassay (ELISA) kit according to the manufacturer's instructions (eBioscience, USA). Briefly, $100 \mu$ of sera were added to each well of a 96-well plate precoated with $100 \mu \mathrm{l} /$ well capture antibody and incubated for $2 \mathrm{~h}$ at $37^{\circ} \mathrm{C}$. The wells were washed and a detection antibody (biotin-conjugated antibody) was added for 1 $\mathrm{h}$ at $37^{\circ} \mathrm{C}$. Wells were washed, and an avidin-horseradish peroxidase (HRP)-labeled $\mathrm{Ab}$ was added for $30 \mathrm{~min}$ at $37^{\circ} \mathrm{C}$. A final wash was performed, and the presence of HRP-labeled Abs was revealed by the addition of tetramethylbenidine (TMB). The reaction was stopped with sulfuric acid $(2 \mathrm{~N})$. Coloration was evaluated using an optical density at $450-570 \mathrm{~nm}$.

\section{Phagocytic function of ON/RAW264.7 cells using fluorescence and FACS}

Microsphere pellets were resuspended in 3\% (w/v) BSA and incubated at $37^{\circ} \mathrm{C}$ for $30 \mathrm{~min}$. Microspheres were sonicated in a bath sonicator at $80 \mathrm{~W}$ for $5 \mathrm{~min}$. The coating of microspheres in BSA ensures that beads remain in a monodispersed state and do not aggregate. OV/RAW264.7 and ON/RAW264.7 cells were plated at $5 \times 10^{5}$ cells/well in 6 -well culture plates (USA) for $24 \mathrm{~h}\left(37^{\circ} \mathrm{C}, 5 \% \mathrm{CO}_{2}\right.$, humidified), and microspheres were added to each well at a final cell to particle ratio of 1:15 $(1 \mu \mathrm{m})$ for approximately $90 \mathrm{~min}$ at $37^{\circ} \mathrm{C}$. The culture media were aspirated, and cells were washed three times with ice-cold PBS to remove excess beads. Cells were observed under fluorescence microscopy to analyze the phagocytosis rate and phagocytosis index or lifted from culture plates by gentle scraping for flow cytometry analyses. Mean fluorescence represents the average fluorescent intensity of all intact cells counted per experiment.
$\boldsymbol{A}$
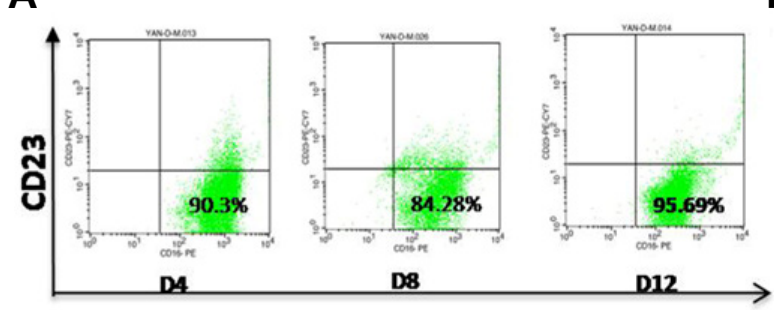

CD16/32

C

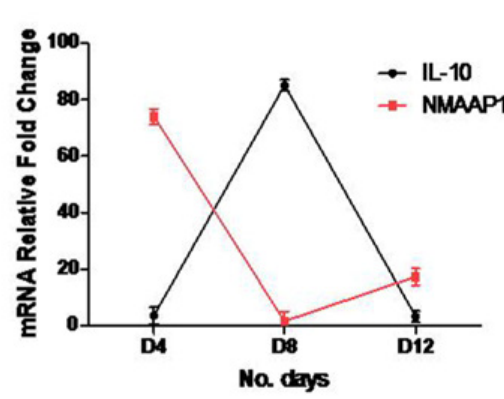

B

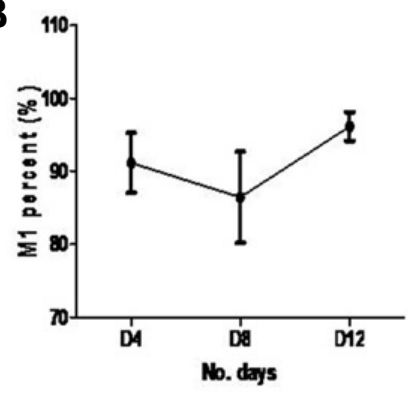

D

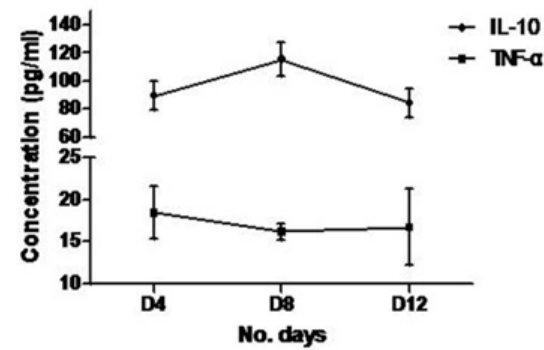

Fig. 1. NMAAP1 expression was associated with the percent of M1 macrophages in BCG-activated peritoneal macrophages. (A) The percent of M1 macrophages in peritoneal macrophages on day 4 , day 8 and day 12 were assessed using FACS. (B) Linear graphs analysis of the percent of M1 macrophages in BCG-activated macrophage. (C) IL-10 and NMAAP1 expression after BCG stimulation on day 4 , day 8 and day 12 was determined using quantitative RTPCR (qPCR). (D) Determination of IL-10 and TNF- $\alpha$ release using ELISA in BCG-stimulated mice sera after day 4 , day 8 and day 12 . Data are represented as mean \pm S.E.M. from at least three independent experiments. 

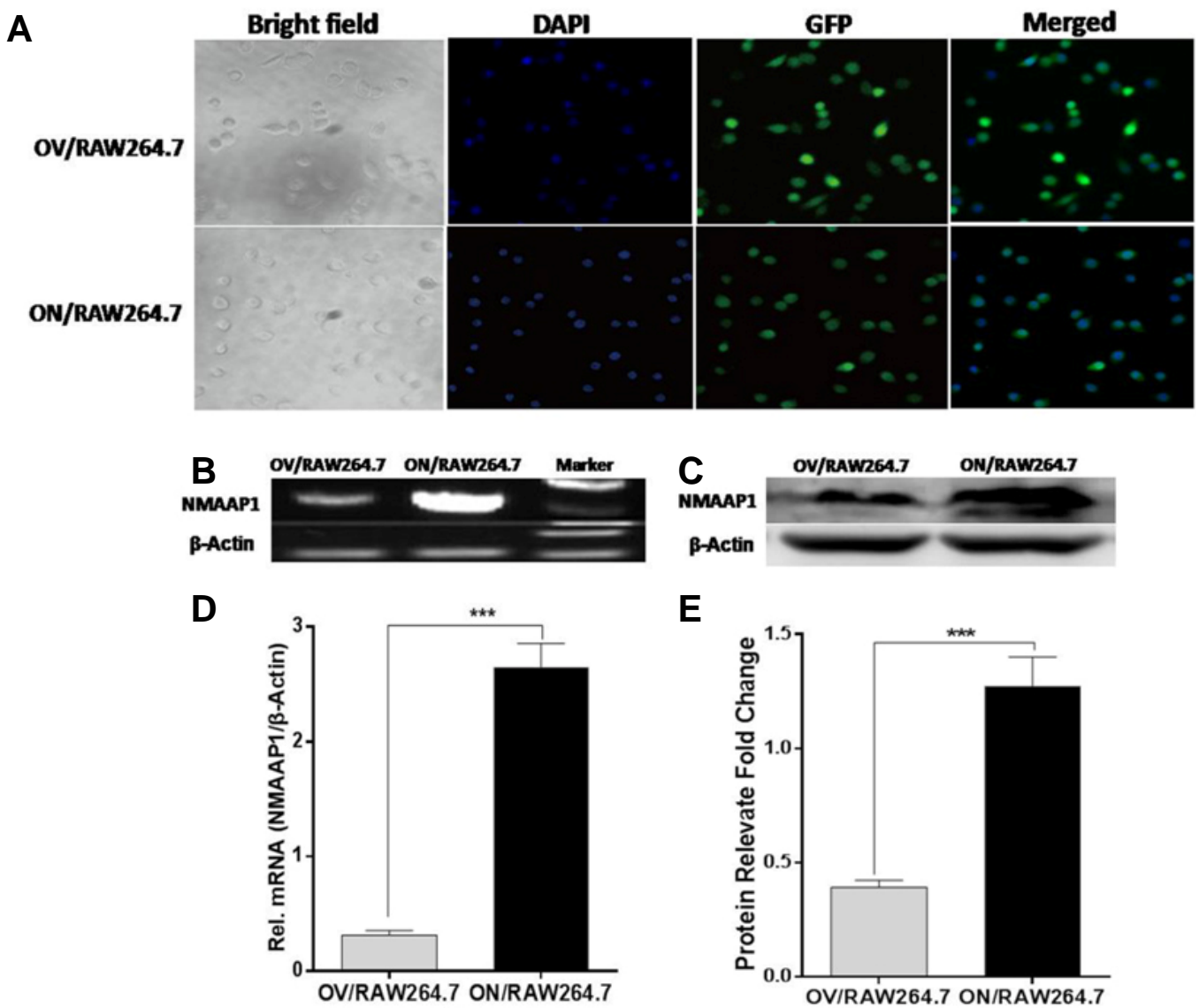

Fig. 2. pIRES2-EGFP/NMAAP1 transfection and transgene expression in RAW264.7 cells, Identification of stable transfectants expressing NMAAP1. (A) RAW264.7 cells were stably transfected with pIRES2-EGFP/NMAAP1 or pIRES2-EGFP/CONTROL. Bright field, DAPI, GFP and DAPI merged with GFP images were taken by a laser-scanning confocal microscope. (B, C) RT-PCR analysis. Expression in OV/RAW264.7 and ON/RAW264.7 cells were detected using RT-PCR. Bar graph displays means \pm SEM of the percentage of NMAAP1/GAPDH ratio for the respective groups. (D, E) Protein expression of NMAAP1 in RAW264.7 cells normalized to $\beta$-Actin as an internal standard: lane 1, pIRES2-EGFP/CONTROL transfected cells; lane 2, pIRES2-EGFP/NMAAP1 transfected cells. Bar graph displays the densitometry results (arbitrary units $[A U]$ ) of the Western blots for the respective groups. Data are represented as mean $\pm S$.E.M. from at least three independent experiments. ${ }^{* \star *} \mathrm{P}<0.001$ (unpaired Student $t$ test. compared with the corresponding control values).

\section{Protein preparation and Western blot analysis}

Various cells were harvested and lysed in lysis buffer (50 $\mathrm{mM}$ Tris- $\mathrm{HCl}, 1 \% \mathrm{NP} 40,150 \mathrm{mM} \mathrm{NaCl}, 1 \mathrm{mM}$ EDTA and 1 $\mathrm{mM}$ PMSF) for $30 \mathrm{~min}$ at $4^{\circ} \mathrm{C}$. Total cell extracts were separated using $12 \%$ SDS/PAGE gels and transferred to PVDF membranes. Membranes were blocked with $3 \%$ BSA and incubated with primary antibodies diluted in blocking solution. The signals were visualized using the chemiluminescent substrate method and the SuperSignal West Pico Kit (Pierce). The $\beta$-actin was used as an internal control to normalize the loading materials.

\section{RESULTS}

\section{NMAAP1 expression coincides with M1 markers in} BCG-activated macrophages

We examined the phenotype of BCG-activated macrophages to delineate the effect of BCG on macrophage biological functions. We detected the expression of $\mathrm{M} 1$ and $\mathrm{M} 2$ cell markers and NMAAP1 levels. CD16/32 (M1) and CD23 (M2) were used to detect the phenotypes of BCG-activated macrophages. The percentage of BCG-activated macrophages that expressed M1 markers increased on day 4 and decreased on day 8 . Mice were challenged with i.p. BCG on day 10 . Therefore, there was an increase in these markers on day 12 (Figs. 1A and 1B).

We next examined whether NMAAP1 was critical in macrophage polarization. We performed quantitative RT-PCR (qPCR) analysis of BCG-activated macrophages (BAMs). NMAAP1 mRNA increased in BAMs with a peak of a $~ 70-$ fold increase on day 4 and decreased in the following 4 days. NMAAP1 mRNA expression increased from day 8 to day 12 and decreased subsequently. NMAAP1 mRNA expression increased slightly on day 12 . There was a similar pattern of changes in the percentage of M1 BAMs. IL-10 mRNA expression increased slowly from day 4 to day 8 and declined rapidly until day 12 . Therefore, the change in IL-10 mRNA expression was opposite to NMAAP1 mRNA expression (Fig. 1C). We performed ELISAs to measure IL-10 and TNF- $\alpha$ release in BCG-stimulated mice sera on day 4 , 8 and 12 (Fig. 1D). 
A
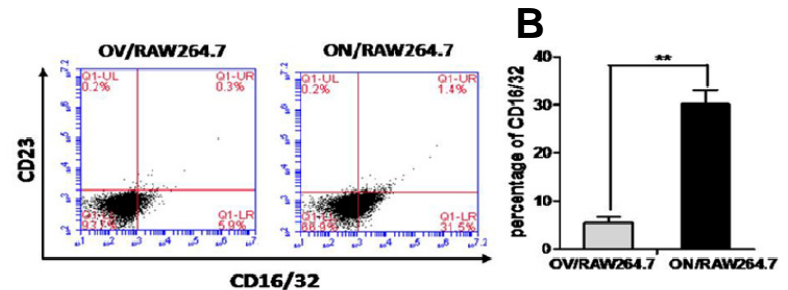

C

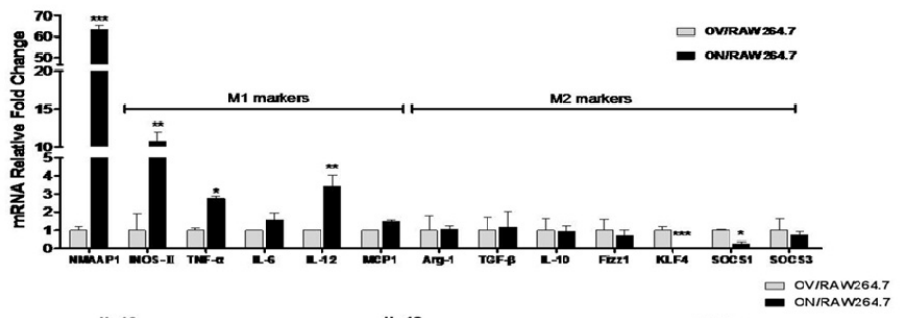

Fig. 3. NMAAP1 altered the gene and protein expression of phenotype-related molecules. The highly expression of NMAAP1 regulated phenotype-specific markers for M1/M2 macrophages. (A, B) The percent of CD16/32 cells in ON/RAW264.7 cells using FACS. (C) M1/M2 genes were evaluated using quantitative real-time RTPCR. (D, E) Release of M1/M2 cytokines was detected using ELISA in $6 \mathrm{~h}, 12 \mathrm{~h}, 24 \mathrm{~h}$ and $48 \mathrm{~h}$ culture supernatants of OV/RAW264.7 and ON/RAW264.7 cells. Data are represented as mean \pm S.E.M. from at least three independent experiments. ${ }^{*} \mathrm{P}<0.05,{ }^{* *} \mathrm{P}<0.01$ and ${ }^{* * *} \mathrm{P}$ $<0.001$ (unpaired Student $t$ test. compared with the corresponding control values).

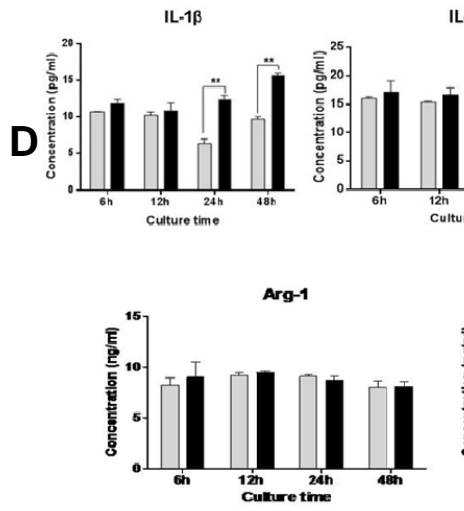

IL-12 TNF-a

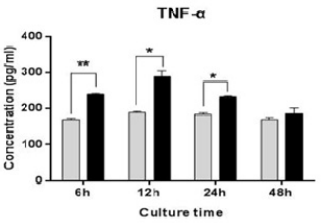

믐 ovrrawasa.7

IL-10

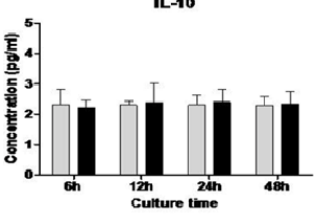

\section{NMAAP1 overexpression in RAW264.7 cells}

Some morphological changes in RAW264.7 were observed after gene transfection. EGFP expression was observed 24 and 48 hours after transfection using fluorescent microscopy, and the transfection efficiency reached peak levels at $72 \mathrm{~h}$. RAW264.7 cells were transfected with pIRES2-EGFP/NMAAP1 or pIRES2-EGFP/CONTROL expression plasmids and selected with G418 as indicated. Stably transfected cells were observed in the same field (Fig. 2A). The results of RT-PCR and Western blot analyses verified that RAW264.7 cells transfected with pIRES2-EGFP/NMAAP1 overexpressed NMAAP1 (ON/RAW264.7) compared to cells transfected with empty pIRES2-EGFP/CONTROL (OV/RAW264.7) (Figs. 2B-2E). The molecular weight of the expressed full-length NMAAP1 was approximately $63 \mathrm{kDa}$, which is consistent with the previously reported molecular weight of NMAAP1 (Zhao et al., 2011).

\section{NMAAP1 overexpression contributes to M1 polarization of} RAW264.7

Classically activated macrophages exhibit a TNF- $\alpha^{\text {high }} i N-$ OS-II ${ }^{\text {high }}$ cytokine profile and exhibit an increased expression of CD16/32 (Herder et al., 2014; Liu et al., 2013). Therefore, we evaluated whether NMAAP1 played a regulatory role in macrophage polarization. We characterized ON/RAW264.7 cells along an M1/M2 spectrum using functional and phenotypic readouts. Cells were stained and analyzed using flow cytometry of CD16/32 and CD23 expression (Fig. 3A). A significantly higher percentage of ON/RAW264.7 cells expressed CD16/32 compared to control cells, but lower CD23 expression was observed $(30.17 \%$ vs. $5.4 \%$, respectively; $p<$
0.01) (Fig. 3B). We quantified the expression of phenotypespecific markers of OV/RAW264.7 and ON/RAW264.7 cells using quantitative real-time RT-PCR analyses to determine the influence of NMAAP1 on $\mathrm{M} 1$ and $\mathrm{M} 2$ gene transcription (Fig. 3C). The M1 markers iNOS, TNF- $\alpha, \mathrm{IL}-6, \mathrm{IL}-12$ and MCP-1 were expressed at higher levels in ON/RAW264.7 cells than OV/RAW264.7 cells, which is consistent with the peritoneal macrophage results. The M2 markers TGF- $\beta$, Arg- 1 , Fizz1 and SOCS3 and the anti-inflammatory cytokine IL-10 were not significantly altered. The expression levels of KLF4 and SOCS1 were markedly lower in ON/RAW264.7 cells than OV/RAW264.7 cells.

TNF- $\alpha$ concentrations in the supernatant of ON/RAW264.7 cells was higher than OV/RAW264.7 cells at $6 \mathrm{~h}, 12 \mathrm{~h}$ and 24 h. ON/RAW264.7 cells produced more IL-1 $\beta$ than OV/RARW 264.7 cells at $24 \mathrm{~h}$ and $48 \mathrm{~h}$. ON/RAW264.7 cells produced slightly higher IL-12 than OV/RARW264.7 cells (Fig. 3D). There were no differences in the levels of the antiinflammatory molecular markers Arg-1 and IL-10 in the supernatants of ON/ RAW264.7 and OV/RAW264.7 cells (Fig. $3 \mathrm{E})$. These results indicate that NMAAP1 regulates some, but not all, M1 genes.

NMAAP1 modulates macrophage-mediated phagocytosis Phagocytosis is the process by which invading pathogens and dead cells are efficiently removed by phagocytic cells, primarily macrophages. Macrophage phagocytosis plays an important role in host defense (Das et al., 2014). The phagocytic activities of OV/RAW264.7 and ON/RAW264.7 cells were measured using fluorescent microspheres after co-incubation 

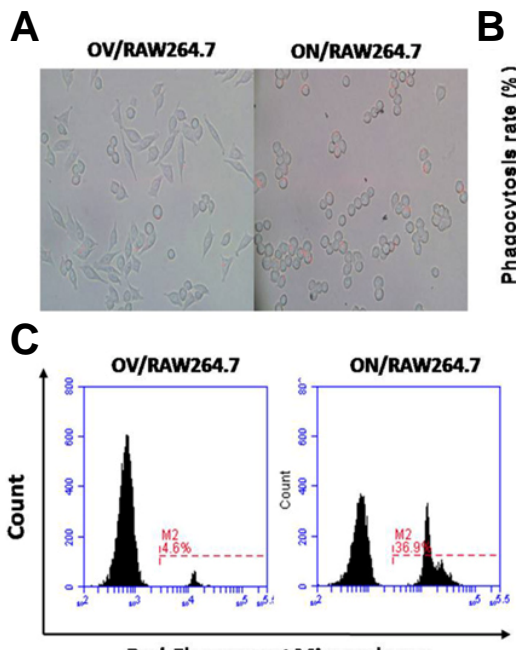

Red-Fluorescent Microspheres
B
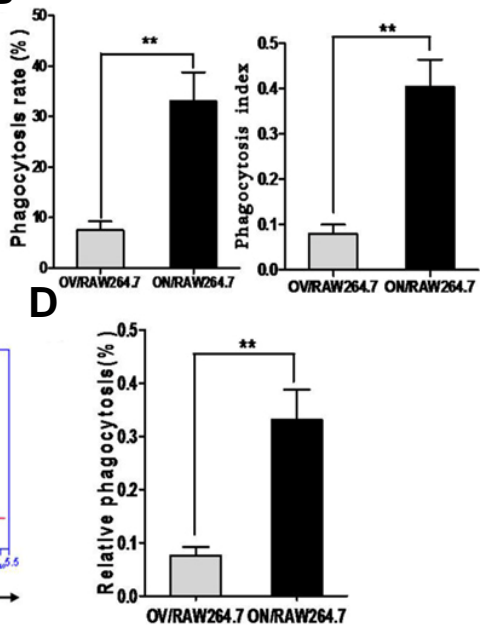

Fig. 4. Macrophage-mediated phagocytosis is modulated by NMAAP1. (A) The images show phagocytosis of fluorescent microspheres by OV/RAW264.7 and ON/RAW264.7 cells incubated with red carboxylatemodified fluorescent beads for $60 \mathrm{~min}$. The cellular uptake of fluorescent beads was determined using fluorescence microscopy (x 40). (B) The phagocytosis rates and phagocytosis index of ON/RAW264.7 cells were obviously increased compared to the OV/RAW264.7 cells $\left({ }^{* *} p<\right.$ 0.01) (C, D) Relative phagocytosis was assayed using flow cytometry as the intensity of fluorescence. Data are represented as mean \pm S.E.M. from at least three independent experiments. ${ }^{* *} \mathrm{P}<0.01$ (unpaired Student $t$ test. compared with the corresponding control values).

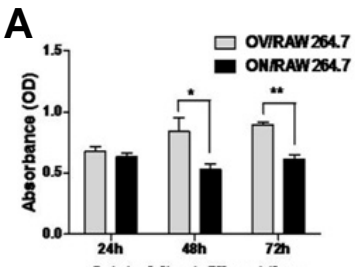

B

cytotoxidity at diflerent time

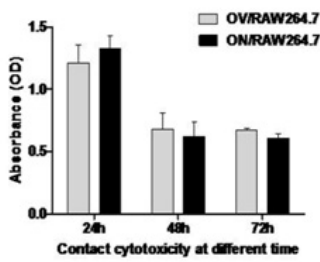

D

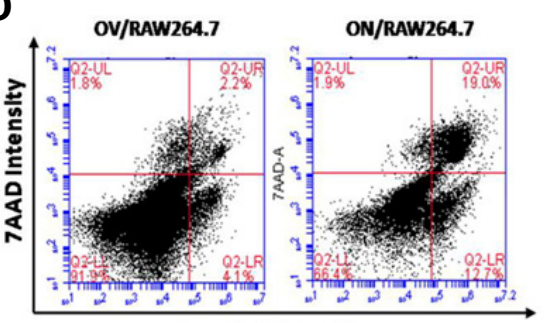

Annexin-Vintensity

C

OV/RAW264.7 + MCA207
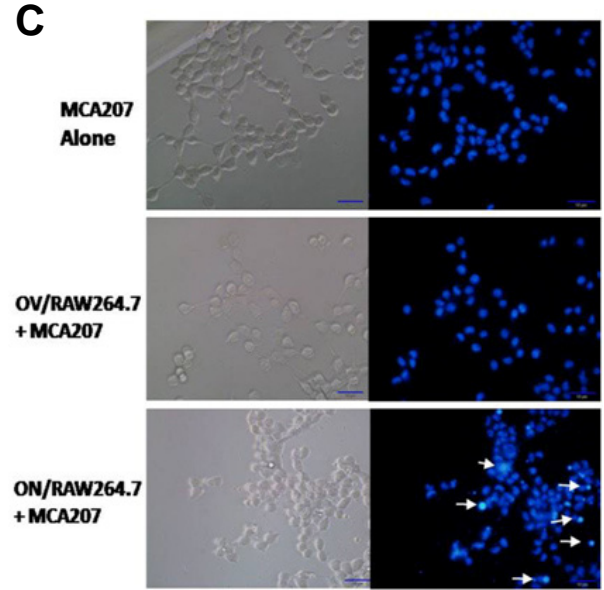

E

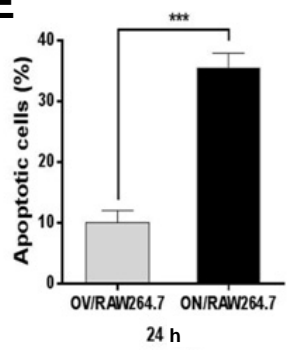

Fig. 5. Tumor cytotoxicity of OV/RAW264.7 and ON/RAW264.7 cells in vitro. (A) Tumor cytoxicity of supernatants of high NMAAP1-expressing RAW264.7 cells in vitro. The supernatant of ON/RAW264.7 cells exhibited significant cytotoxicity compared to the control group at $48 \mathrm{~h}$ and $72 \mathrm{~h}$. (B) Contact cytotoxicity of paraformaldehyde-fixed macrophages. (C) Detection of cell apoptosis using immunofluorescence with Hoechst 33342 staining. (D) NMAAP1 plays an antioncogenic role in cytokines-induced MCA207 sarcoma cell apoptosis. MCA207 sarcomas cells cultured with supernatants of OV/RAW264.7 or ON/RAW264.7, and apoptosis was evaluated using 7AAD/Annexin-V dual staining at $24 \mathrm{~h}$. Cells in the upper right (UR) portion are late-stage apoptotic cells, upper left (UL) are necrotic cells, and cells in the lower left (LL) and lower right (LR) portions are viable and early apoptotic cells, respectively. (E) Bar graph showing the percentage of apoptotic MCA207 cells using flow cytometry. Data are represented as mean \pm S.E.M. from at least three independent experiments. ${ }^{*} \mathrm{P}$ $<0.05,{ }^{* *} P<0.01$ and ${ }^{* * *} P<0.001$ (unpaired Student $t$ test. compared with the corresponding control values). for $2 \mathrm{~h}$. Phagocytosis rates and phagocytosis index of OV/RAW264.7 and ON/RAW264.7 cells were quantified using fluorescence microscopy (Figs. 4A and 4B) and flow cytometry (Figs. 4C and 4D). OV/RAW264.7 and ON/RAW264.7 cells efficiently phagocytize fluorescent microspheres. However, ON/RAW264.7 cells exhibited a significantly higher phagocytosis rate and phagocytosis index against xenogeneic target microspheres than OV/RAW264.7 cells.

\section{NMAAP1 promotes tumoricidal activities of RAW264.7}

Cytotoxicity assays were performed via the culture of tumor cells with supernatants collected from OV/RAW264.7 and
ON/RAW264.7 cultures to determine the influence of NMAAP1 on the tumoricidal activity of macrophages. ON/RAW264.7 cells presented a more prominent cytotoxicity effect for the inhibition of MCA207 tumor cell viability than OV/RAW264.7 cells (Fig. 5A). We measured the contact-dependent tumoricidal function and excluded the possible effect of soluble factors using paraformaldehyde (PFA)-fixed macrophages. PFA-fixed OV/RAW264.7 and ON/RAW264.7 cells did not significantly alter the cytotoxicity of cells co-cultured with MCA207 cells at $24 \mathrm{~h}, 48 \mathrm{~h}$ and $72 \mathrm{~h}$ (Fig. $5 B$ ). Tumor cell death was measured using morphological analyses (Hoechst 33342) and flow cytometry to further confirm these results. Figure $5 \mathrm{C}$ shows that most cells from the control 
group (MAC207 alone and OV/RAW264.7 + MCA207) contained large and regular nuclei. Clear evidence of chromatin condensation and a clear decrease in cell size was observed in cells cultured for $48 \mathrm{~h}$ in the supernatant of ON/RAW264.7 cells cultured for $24 \mathrm{~h}$ (white arrow). Cell death was also measured using the fluorescent dye Annexin V-FITC, which binds phosphatidylserine residues that are redistributed from the inner to the outer leaflet of the cell membrane as an early event in apoptosis. After the loss of membrane integrity, 7AAD enters the cell and intercalates into DNA. Figures $5 D$ and $5 E$ show the percentages of Annexin V-stained and 7AAD-stained cells in response to the supernatants of OV/RAW264.7 and ON/RAW264.7 cells (24 h). The supernatant of ON/RAW264.7 cell culture (48 h) on MCA207 culture for $24 \mathrm{~h}$ induced a greater population of apoptotic cells than the OV/RAW264.7 group $(33.6 \%$ vs. $8.1 \%)$. These data suggest that NMAAP1 exerts prominent effect on the modulation of macrophage-mediated cytotoxicity, which may occur via NMAAP1 induction of macrophage secretion of more inflammatory cytokines.

\section{DISCUSSION}

Macrophages (Mdp) are a heterogeneous cell population that are involved in diverse physiological processes, including antimicrobial defense, wound resolution, inflammation, tissue remodeling and the promotion of tumor growth (Epelman et al., 2014; Wynn et al., 2013). Macrophages achieve high tumoricidal activity after activation by some stimuli, such as BCG. BCG is the attenuated (virulence-reduced) live bovine tuberculosis bacillus, Mycobacterium bovis, which has lost its virulence in humans. Macrophages recognize BCG through Toll-like receptor 2 (TLR2), Toll-like receptor 9 (TLR9) and possibly Tolllike receptor 4 (TLR4) and become activated. Activated macrophages have been used for the treatment of some bladder cancers for more than two decades (Huang et al., 2015; Liu et al., 2014), and they exhibit antitumor effects via direct cell-tocell contact (Zhang et al., 2007). Macrophages with strong microbicidal and tumoricidal activities are generally regarded as M1 macrophages, with characteristics of TNF- $\alpha, \mathrm{IL}-1 \beta$, inducible nitric oxide synthase (iNOS or NOS2), and reactive nitrogen and oxygen intermediates production and promotion of Th1 responses (Biswas et al., 2012; Sica and Mantovani, 2012). Our results demonstrated that macrophages from BCGinjected mice exhibited an M1 polarized phenotype. However, previous reports demonstrated that some components of Mycobacterium tuberculosis, such as lipoproteins, DnaK and PPE18, interact with TLR2 and activate IL-10 induction in macrophages and polarize macrophages to the M2-like phenotype (Lopes et al., 2014; Nair et al., 2009; Pecora et al., 2006). One reason for these differences may be that the pathogenic mycobacterium bovis strains differ in their ability to modulate the polarization of macrophages. The B2 and H37Rv strains synergistically induce M1 polarization, and the MP287/03 strain expresses high levels of Arg-1, which lead to lower levels of NO production (Andrade et al., 2012). Another reason may be that different components of attenuated and viable $\mathrm{M}$. tuberculosis induce the absolute opposite polarization of macrophages. Lipoproteins and the MHC class II inhibition of M. tuberculosis may be destroyed after attenuation, but other components that promote M1 polarization of macrophages remain (Pai et al., 2014). The components of BCG that promote M1 polarization require more experiments to support these hypotheses.

The expression of many proteins was upregulated in BCGactivated macrophages, which would play important roles in the tumoricidal effect or modulatory activities. The novel protein NMAAP1 was specifically expressed on BCG-stimulated macrophages, and it played a crucial role in the regulation of $M 1$ macrophage polarization. BCG-activated macrophages maintained NMAAP1 expression during a state of high activity in M1-polarized cells, which facilitated M1 gene expression. Upregulated NMAAP1 expression in RAW264.7 cells increased the expression of M1 markers (e.g., CD16/32, iNOS, TNF- $\alpha$, IL6 , IL-12, MCP-1 and IL-1 $\beta$ ), and decreased or did not affect M2 markers (e.g., KLF4, SOCS1, IL-10, SOCS3, Fizz1 and Arg-1) (Labonte et al., 2014).

BCG-activated macrophages kill tumor cells through cell-tocell contact and the secretion of tumoricidal mediators, such as iNOS, TNF- $\alpha$, INF- $\gamma$, IL-2, IL-6 and IL-12, which induce macrophage activation and cytotoxic activity, and IL-10 plays a negative role in BCG-induced macrophage cytotoxicity (Luo and Knudson, 2010). However, knowledge of the mechanism of cell-cell contact-mediated killing is limited. BCG infection may upregulate adhesion molecules, such as lymphocyte function-associated antigen-1 (LFA-1) or apoptosis-inducing molecules, such as Fas ligand and TRAIL on macrophages (DesJardin et al., 2002; Diehl et al., 2004). Soluble factors released from BCG-activated human monocytes/macrophages contributed to the total killing of bladder cancer UCRU-BL-17 cells more than effector-target cell contact (Pryor et al., 1995). NMAAP1-transfected RAW264.7 cells exhibited profound toxic effects on tumor cells, especially the induction of tumor cell apoptosis. Cytotoxicity was also enhanced through the secretion of iNOS, TNF- $\alpha$, and IL-6. However, tumoricidal effects on tumor cells through cell-to-cell contact were not detected. This result may be because NMAAP1 could not regulate the tumoricidal membrane protein expression on macrophages. Activated macrophages engulf tumor cells via antibody-mediated phagocytosis (Overdijk et al., 2015). The effect of increased phagocytic ability suggests that NMAAP1 plays an important role in the clearing of tumor cells and invading pathogens (Das et al., 2014), and it may enhance the expression of some adhesive molecules and IgG receptors (DesJardin et al., 2002; Shi et al., 2015).

NMAAP1 is a membrane-associated protein that contains an open-reading frame (ORF) that encodes a peptide of $555 \mathrm{ami}-$ no acids and a small portion of the MAB-21 domain that is expressed in a wide range of tissues (Zhao et al., 2011). Members of the MAB-21 family control cellular differentiation in $C$. elegans, Danio rerio, and Mus musculus (Heanue and Pachnis, 2006; Yamada et al., 2004).

NMAAP1 bears a similar amino acid sequence to the human protein kiaa1754, which is a regulatory protein for inositol 1,4,5trisphosphate-receptor activity. The kiaa1754 protein is a membrane-associated IP3R-binding protein that physiologically binds IP3R to regulate calcium release and neuronal process outgrowth in neuronal cell lineages (van Rossum et al., 2006). We hypothesized that NMAAP1 shared a regulatory activity on IP3R and played an important role via IP3R-mediated calcium signaling to trigger the differentiation of certain macrophage subpopulations and prompt phagocytic and tumoricidal activities of macrophage.

Various factors, including cytokines/chemokines, hormones, and external biomechanical stimuli affect macrophage phenotype. The mechanism of actions of these factors is related to calcium signaling (Collins and Meyer, 2009; Evans and Fake, 2007; Través et al., 2013). $\mathrm{Ca}^{2+}$ is a highly versatile intracellular signaling messenger that regulates many different cellular processes during the entire cell lifespan, such as cell differentiation, 
proliferation, secretion and synaptic plasticity. Recent reports demonstrate that several molecules, such as DANGER (van Rossum et al., 2006), cytochrome c (Boehning et al., 2003; 2005), polyglutamine expanded huntingtin $\left(\mathrm{Htt}^{\mathrm{exp}}\right)$ and huntingtin-associated protein-1A (Tang et al., 2003), strictly regulate $\mathrm{Ca}^{2+}$ release activity via binding to IP3R in physiological and pathological situations.

These results demonstrated that BCG increased NMAAP1 expression in macrophages, and NMAAP1 may promote the polarization of M1 macrophages, which exhibit tumoricidal activities through the secretion of inducible mediators.

Note: Supplementary information is available on the Molecules and Cells website (www.molcells.org).

\section{ACKNOWLEDGMENTS}

The authors thank Prof. Yi Li for helpful discussions. This work was supported by the Project of National Natural Science Foundation of China (Nos. 81401282), Health Department of Jilin Province (Nos. 2012z056) and the Jilin Province Science Foundation for Youths (Nos.20140520011J).

\section{REFERENCES}

Andrade, M.R., Amaral, E.P., Ribeiro, S.C., Almeida, F.M., Peres, T.V., Lanes, V., D'Império-Lima, M.R., and Lasunskaia, E.B. (2012). Pathogenic Mycobacterium bovis strains differ in their ability to modulate the proinflammatory activation phenotype of macrophages. BMC Microbiol. 12, 166.

Baldessari, D., Badaloni, A., Longhi, R., Zappavigna, V., and Consalez, G.G. (2004). MAB21L2, a vertebrate member of the Male-abnormal 21 family, modulates BMP signaling and interacts with SMAD1. BMC Cell Biol. 5, 48.

Benoit, M., Desnues, B., and Mege, J.L. (2008). Macrophage polarization in bacterial infections. J. Immunol. 181, 3733-3739.

Biswas, S.K., and Mantovani, A. (2012). Orchestration of metabolism by macrophages. Cell Metab. 15, 432-437.

Boehning, D., Patterson, R.L., Sedaghat, L., Glebova, N.O., Kurosaki, T., and Snyder, S.H. (2003). Cytochrome c binds to inositol $(1,4,5)$ trisphosphate receptors, amplifying calcium-dependent apoptosis. Nat Cell Biol. 5, 1051-1061.

Boehning, D., van Rossum, D.B., Patterson, R.L., and Snyder, S.H. (2005). A peptide inhibitor of cytochrome c/inositol 1,4,5trisphosphate receptor binding blocks intrinsic and extrinsic cell death pathways. Proc. Natl. Acad. Sci. USA 102, 1466-1471.

Chan, T., Pek, E.A., Huth, K., and Ashkar AA (2011). CD4(+) T-cells are important in regulating macrophage polarization in C57BL/6 wild-type mice. Cell Immunol. 266, 180-186.

Collins, S.R., and Meyer, T. (2009). Calcium flickers lighting the way in chemotaxis? Dev. Cell. 16, 160-161.

Das, R., Ganapathy, S., Settle, M., and Plow, E.F. (2014). Plasminogen promotes macrophage phagocytosis in mice. Blood 124, 679-688.

DesJardin, L.E., Kaufman, T.M., Potts, B., Kutzbach, B., Yi, H., and Schlesinger, L.S. (2002). Mycobacterium tuberculosis-infected human macrophages exhibit enhanced cellular adhesion with increased expression of LFA-1 and ICAM-1 and reduced expression and/or function of complement receptors, FcgammaRII and the mannose receptor. Microbiology 148, 3161-3171.

Diehl, G.E., Yue, H.H., Hsieh, K., Kuang, A.A., Ho, M., Morici, L.A., Lenz, L.L., Cado, D., Riley, L.W., and Winoto, A. (2004). TRAIL$\mathrm{R}$ as a negative regulator of innate immune cell responses. Immunity $21,877-889$.

Epelman, S., Lavine, K.J., and Randolph, G.J. (2014). Origin and functions of tissue macrophages. Immunity 41, 21-35.

Evans, J.H., and Falke, J.J. (2007). $\mathrm{Ca}^{2+}$ influx is an essential component of the positive-feedback loop that maintains leadingedge structure and activity in macrophages. Proc. Natl. Acad. Sci. USA 104, 16176-16181.

Gordon, S., and Martinez, F.O. (2010). Alternative activation of macrophages: mechanism and functions. Immunity 32, 593-604

Heanue, T.A., and Pachnis, V. (2006). Expression profiling the de- veloping mammalian enteric nervous system identifies marker and candidate Hirschsprung disease genes. Proc. Natl. Acad. Sci. USA 103, 6919-6924.

Herder, V., Iskandar, C.D., Kegler, K., Hansmann, F., Elmarabet, S.A., Khan, M.A., Kalkuhl, A., Deschl, U., Baumgärtner, W., Ulrich, R., et al. (2014). Dynamic changes of microglia/macrophage M1 and M2 polarization in theiler's murine encephalomyelitis. Brain Pathol. doi: 10.1111/bpa.12238. [Epub ahead of print]

Ho, S.H., So, G.M., and Chow, K.L. (2001). Postembryonic expression of Caenorhabditis elegans mab-21 and its requirement in sensory ray differentiation. Dev. Dyn. 221, 422-430

Huang, P., Ma, C., Xu, P., Guo, K., Xu, A., and Liu, C. (2015).Efficacy of intravesical Bacillus Calmette-Guérin therapy against tumor immune escape in an orthotopic model of bladder cancer. Exp. Ther. Med. 9, 162-166.

Kang, B.N., Ahmad, A.S., Saleem, S., Patterson, R.L., Hester, L., Doré, S., and Snyder, S.H. (2010). Death-associated protein kinase-mediated cell death modulated by interaction with DANGER. J. Neurosci. 30, 93-98.

Labonte, A.C., Tosello-Trampont, A.C., and Hahn, Y.S. (2014). The role of macrophage polarization in infectious and inflammatory diseases. Mol. Cells 37, 275-285.

Liu, C., Li, Y., Yu, J., Feng, L., Hou, S., Liu, Y., Guo, M., Xie, Y., Meng, J., Zhang, H., et al. (2013). Targeting the shift from M1 to M2 macrophages in experimental autoimmune encephalomyelitis mice treated with fasudil. PLoS One 8, e54841.

Liu, X., Dowell, A.C., Patel, P., Viney, R.P., Foster, M.C., Porfiri, E., James, N.D., and Bryan, R.T. (2014). Cytokines as effectors and predictors of responses in the treatment of bladder cancer by bacillus Calmette-Guérin. Future Oncol. 10, 1443-1456.

Lodillinsky, C., Langle, Y., Guionet, A., Góngora, A., Baldi, A., Sandes, E.O., Casabé, A., and Eiján, A.M. (2010). Cytokines as effectors and predictors of responses in the treatment of bladder cancer by bacillus Calmette-Guérin. PLoS One 5, e13571.

Lopes, R.L., Borges, T.J., Araújo, J.F., Pinho, N.G., Bergamin, L.S., Battastini, A.M., Muraro, S.P., Souza, A.P., Zanin, R.F., and Bonorino, C. (2014). Extracellular mycobacterial DnaK polarizes macrophages to the M2-like phenotype. PLoS One 9, e113441.

Luo, Y., and Knudson, M.J. (2010). Mycobacterium bovis bacillus Calmette-Guérin-induced macrophage cytotoxicity against bladder cancer cells. Clin. Dev. Immunol. 2010, 357591.

Luo, Y., Yamada, H., Evanoff, D.P., and Chen, X. (2006). Role of Th1-stimulating cytokines in bacillus Calmette-Guérin(BCG)induced macrophage cytotoxicity against mouse bladder cancer MBT-2 cells. Clin. Explmmunol. 146, 181-188.

Nair, S., Ramaswamy, P.A., Ghosh, S., Joshi, D.C., Pathak, N., Siddiqui, I., Sharma, P., Hasnain, S.E., Mande, S.C., and Mukhopadhyay, S. (2009). The PPE18 of Mycobacterium tuberculosis interacts with TLR2 and activates IL-10 induction in macrophage. J. Immunol. 83, 6269-6281.

Overdijk, M.B., Verploegen, S., Bögels, M., van Egmond, M., Lammerts van Bueren, J.J., Mutis, T., Groen, R.W., Breij, E., Martens A.C., Bleeker, W.K., et al. (2015). Antibody-mediated phagocytosis contributes to the anti-tumor activity of the therapeutic antibody daratumumab in lymphoma and multiple myeloma. MAbs. 7, 311-321.

Pai, R.K., Pennini, M.E., Tobian, A.A., Canaday, D.H., Boom, W.H., and Harding, C.V. (2014). Prolonged toll-like receptor signaling by Mycobacterium tuberculosis and its 19-kilodalton lipoprotein inhibits gamma interferon-induced regulation of selected genes in macrophages. Infect. Immun. 72, 6603-6614.

Park, H.G., Lee, Y.R., Jun, J.K., and Lee, W.L. (2014). Exercise training is more effective than resveratrol supplementation on alleviation of inflammation in peritoneal macrophages of high fat diet mice. J. Exerc. Nutrition Biochem. 18, 79-87.

Pecora, N.D., Gehring, A.J., Canaday, D.H., Boom, W.H., and Harding, C.V. (2006). Mycobacterium tuberculosis LprA is a lipoprotein agonist of TLR2 that regulates innate immunity and APC function. J. Immunol. 177, 422-429.

Pryor, K., Goddard, J., Goldstein, D., Stricker, P., Russell, P., Golovsky, D., and Penny, R. (1995). Bacillus Calmette-Guerin (BCG) enhances monocyte- and lymphocyte-mediated bladder tumour cell killing. Br J. Cancer 71, 801-807.

Shi, Y., Fan, X., Deng, H., Brezski, R.J., Rycyzyn, M., Jordan, R.E., Strohl, W.R., Zou, Q., Zhang, N., and An, Z. (2015). 
Trastuzumab triggers phagocytic killing of high HER2 cancer cells in vitro and in vivo by interaction with $\mathrm{Fc \gamma}$ receptors on macrophages. J. Immunol. 194, 4379-4386.

Sica, A., and Mantovani, A. (2012). Macrophage plasticity and polarization: in vivo veritas. J. Clin. Invest. 122, 787-795

Sica, A., Invernizzi, P., and Mantovani, A. (2014). Macrophage plasticity and polarization in liver homeostasis and pathology. Hepatology 59, 2034-2042.

Svatek, R.S., Zhao, X.R., Morales, E.E., Jha, M.K., Tseng, T.Y., Hugen, C.M., Hurez, V., Hernandez, J., and Curiel, T.J. (2015). Sequential intravesical mitomycin plus Bacillus Calmette-Guérin for non-muscle-invasive urothelial bladder carcinoma: translational and phase I clinical trial. Clin. Cancer Res. 21, 303-311.

Tang, T.S., Tu, H., Chan, E.Y., Maximov, A., Wang, Z., Wellington, C.L., Hayden, M.R., and Bezprozvanny, I. (2003). Huntingtin and huntingtin-associated protein 1 influence neuronal calcium signaling mediated by inositol- $(1,4,5)$ triphosphate receptor type 1. Neuron 39, 227-239

Thompson, D.B., Siref, L.E., Feloney, M.P., Hauke, R.J., and Agrawal, D.K .(2015). Immunological basis in the pathogenesis and treatment of bladder cancer. Exp. Rev. Clin. Immunol. 11, 265-279.

Través, P.G., Pimentel-Santillana, M., Carrasquero, L.M., PérezSen, R., Delicado, E.G., Luque, A., Izquierdo, M., Martín-Sanz, P., Miras-Portugal, M.T., and Boscá, L. (2013). Selective impairment of P2Y signaling by prostaglandin E2 in macrophages: implications for Ca2+-dependent responses. J. Immunol. 190, 4226-4235

van Rossum, D.B., Patterson, R.L., Cheung, K.H., Barrow, R.K.,
Syrovatkina, V., Gessell, G.S., Burkholder, S.G., Watkins, D.N., Foskett, J.K., and Snyder, S.H. (2006). DANGER, a novel regulatory protein of inositol 1,4,5-trisphosphate-receptor activity. J. Biol. Chem. 281, 37111-37116.

Villalta, S.A., Nguyen, H.X., Deng, B., Gotoh, T., and Tidball, J.G (2009). Shifts in macrophage phenotypes and macrophage competition for arginine metabolism affect the severity of muscle pathology in muscular dystrophy. Hum. Mol. Genet. 18, 482-496.

Wang, N., Liang, H., and Zen, K. (2014). Molecular mechanisms that influence the macrophage $\mathrm{m} 1-\mathrm{m} 2$ polarization balance. Front Immunol. 5, 614

Wong, S.C., Puaux, A.L., Chittezhath, M., Shalova, I., Kajiji, T.S., Wang, X., Abastado, J.P., Lam, K.P., and Biswas, S.K. (2010). Macrophage polarization to a unique phenotype driven by $B$ cells. Eur. J. Immunol. 40, 2296-2307.

Wynn, T.A., Chawla, A., and Pollard, J.W. (2013). Macrophage biology in development, homeostasis and disease. Nature 496 445-455.

Yamada, R., Mizutani-Koseki, Y., Koseki, H., and Takahashi, N. (2004). Requirement for Mab21l2 during development of murine retina and ventral body wall. Dev Biol 274, 295-307.

Zhang, L., Lun, Y., Yan, D., Yu, L., Ma, W., Du, B., and Zhu, X (2007). Proteomic analysis of macrophages: a new way to identify novel cell-surface antigens. J. Immunol. Methods 321, 80-85.

Zhao, X., Zhang, L., Yan, D., Feng, X., Chu, D., Li, B., Du, B., and Zhu, X. (2011). Phylogenetic analysis and prokaryotic expression of NMAAP1 derived from BCG-activated murine macrophages. Comp. Immunol. Microbiol. Infect. Dis. 34, 123-128. 